\title{
Enhanced Self-confidence in Young Adolescents with Direct Partial Composite Veneer: A Case Series
}

\author{
Revina Ester Iriani Marpaung and Adioro Soetojo \\ Department of Conservative Dentistry, Faculty of Dental Medicine, Universitas Airlangga, Surabaya, Indonesia
}

\begin{abstract}
Dental esthetic problems in young adolescents such as undesirable tooth shape, color, size and position may affect psychosocial interaction and self-confidence of a person, thus the need for dental treatment. Recent composite resin materials offer a vast range of shades that perfectly emulate natural tooth. The aim of this procedure is to improve the appearance of the teeth using direct partial composite veneer due to its minimally invasive and time efficiency characteristics.
\end{abstract}

Key Words: self-confidence, adolescent, direct veneer

\section{INTRODUCTION}

Dental esthetics has been proven to play a significant impact on an individual's psychosocial well-being. Dentition, the arrangement and condition of the teeth, is a crucial factor in smile creation, hence, a person's facial expression. Individuals who are dissatisfied with their dental appearance might feel the urge to conceal their mouth or refrain from social interaction. Self-perceived satisfaction in dental esthetics has a positive impact on a person's social and psychological behavior and self confidence. ${ }^{1}$ Individuals with low self-esteem rarely smile, have reported being teased about the appearance of their teeth, and believed that straight teeth improve one's popularity and success in life. ${ }^{1}$

Veneers are suitable for esthetics and conservative dental restorations. They can be divided into two (2) categories directly and indirectly fabricated.

Direct veneers can be described as a layer of tooth material, in this case a composite, applied on the tooth surface for esthetics. This treatment is indicated for damaged, defective and malformed facial surfaces, discolored facial surfaces, and for discolored restorations. Direct veneer has the advantage of being more practical because it can be done on a single session, is useful for localized defects, and is economical. ${ }^{2}$ Direct veneers can be divided into two (2) categories based on tooth preparation: direct partial veneers; and direct full veneers. Direct partial veneers are ideally carried out when there are small localized intrinsic discolorations or defects that are surrounded by healthy enamel. Full direct veneers are indicated to restore generalized defects or areas of intrinsic staining involving most of the facial surface of the tooth. ${ }^{3}$ 


\section{CASE 1}

A 23-year-old male patient with fracture and spacing in the upper front teeth region, with esthetic complaints, was referred to Airlangga Dental Hospital. The patient did not feel confident of his dental appearance and encountered difficulties in finding employment due to his unattractive dental esthetic. Intraoral examination revealed the presence of diastema between maxillary central incisors due to misaligned tooth \#8 and distal-incisal edge fractures of maxillary right central incisor. No dental caries were observed (Figure 1).

Clinical examination showed that there was a gap between the maxillary central incisors and a fracture on distal-incisal edge \#8. The shape of the lateral incisors looked less favorable (Figure 1).

A treatment plan was made using Keynote Application (Apple Inc. United States) to prepare a simple digital smile design which will be used as guide for the diagnostic wax up. (Figure 2). It was noted from the digital design that four of the anterior teeth needed treatment to achieve the ideal smile.

After the isolation with rubber dam, shade selection was made using the button shade technique. (Figure 3) Polymerized composite was used to compare the surrounding dentition.

After shade selection, the labial and palatal surfaces of the teeth were slightly roughened with abrasive disc (Soft-Lex ${ }^{\mathrm{TM}}$ Contouring and Polishing Disc, 3M ESPE,

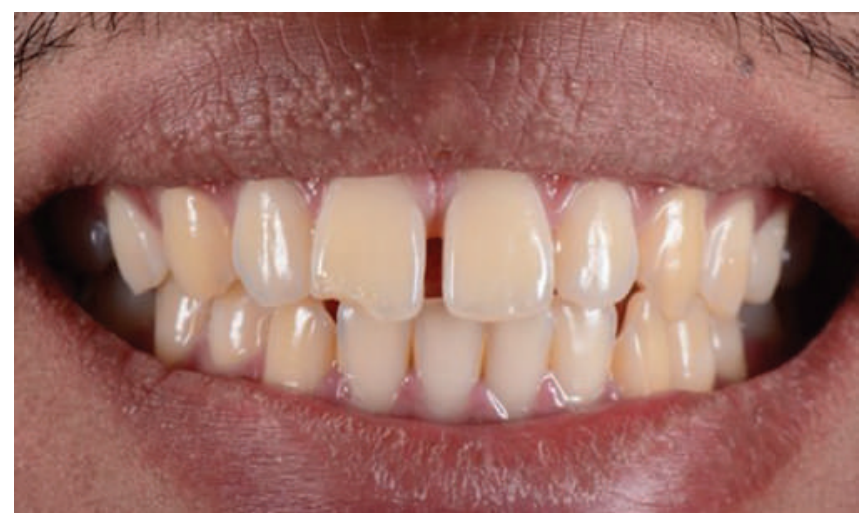

Figure 1. Preoperative look.

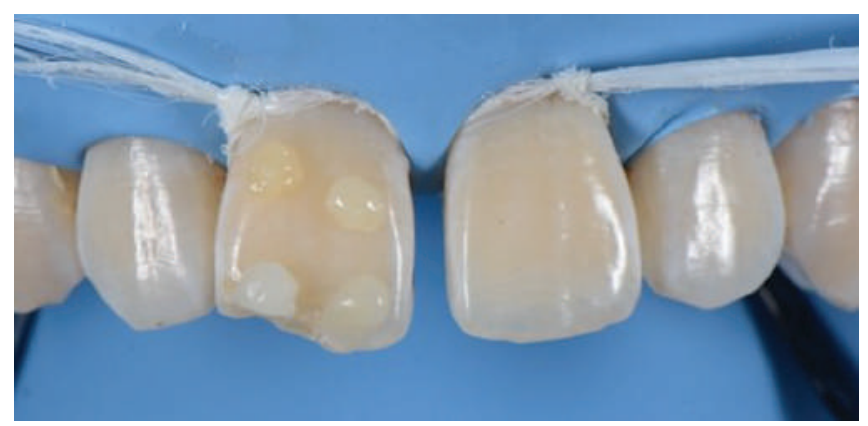

Figure 3. Composite Shades Selection.
United States). Beveling was done on incisal edge \#8 and the misaligned tooth corrected. (Figure 4)

Etching was done with $37 \%$ phosphoric acid for 30 seconds, followed with thorough washing for 20 seconds and then, was air-dried. (Figure 5A)

A thin layer of Universal Adhesive Prime\&Bond universal $^{\text {TM }}$ was applied on circular motion using a microbrush. Once the liquid has penetrated, the air was gently dried to remove excess solvent before polymerization using light-cure technique. (Figure 5B)

Palatal guide was made to determine the proximal lines of the interdental space and the correct position of the incisal edge. A thin layer of enamel shade A2 (Palfique LX5, Tokuyama Dental, Tokyo, Japan) was applied on the silicone index to create the palatal walls. A sectional matrix was then placed on the interdental central incisors with a thin layer of enamel shade to make a proximal wall. (Figure 6)

Dentin layering was carried out with dentin shade UD3 (Enamel plus HRi, Micerium, Avegno, Genova, Italy) and surface enamel layering with enamel shade A2 (Palfique LX5, Tokuyama Dental, Tokyo, Japan).

Facial surface was contoured with abrasive discs (SoftLex ${ }^{\mathrm{TM}}$ Contouring and Polishing Disc, 3M ESPE, United States). Proximal finishing was achieved sequentially, using interdental strips. Enhance ${ }^{\varpi} \mathrm{PoGo}^{\varpi}$ system was used for final polishing. (Figure 7) Final result was achieved (Figure 8A). After one week, the restoration was perfectly integrated and patient had no complaint (Figure 8B).

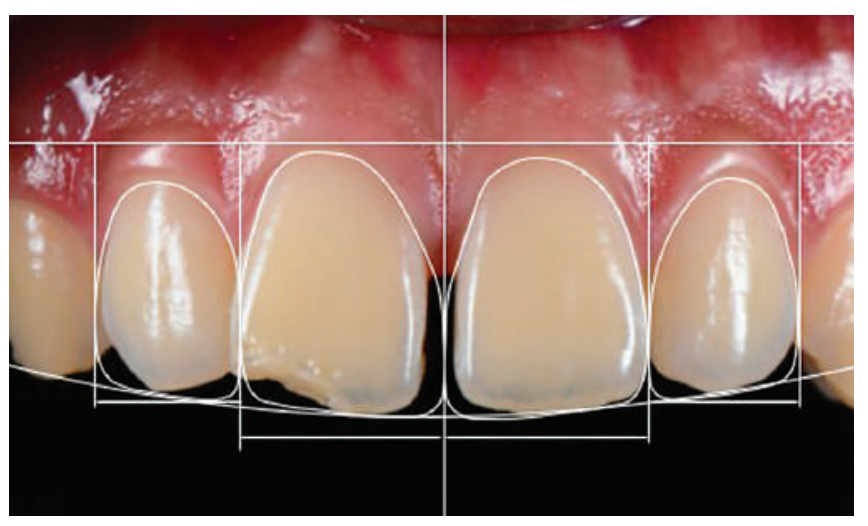

Figure 2. Digital Smile Design with Keynote.

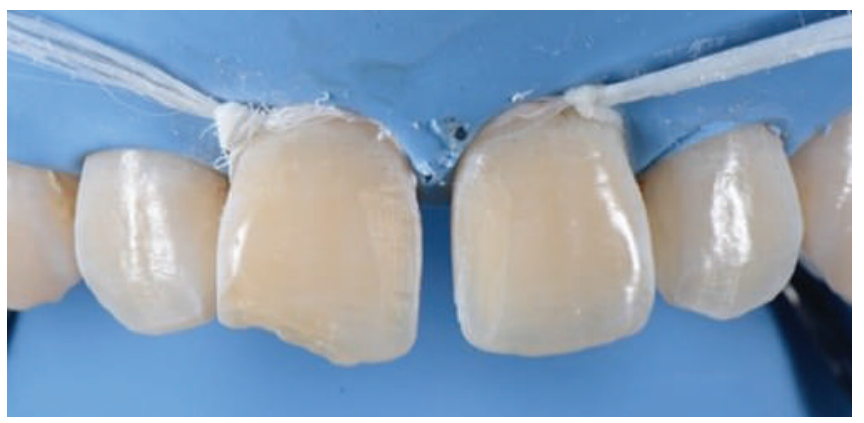

Figure 4. Tooth Preparation. 

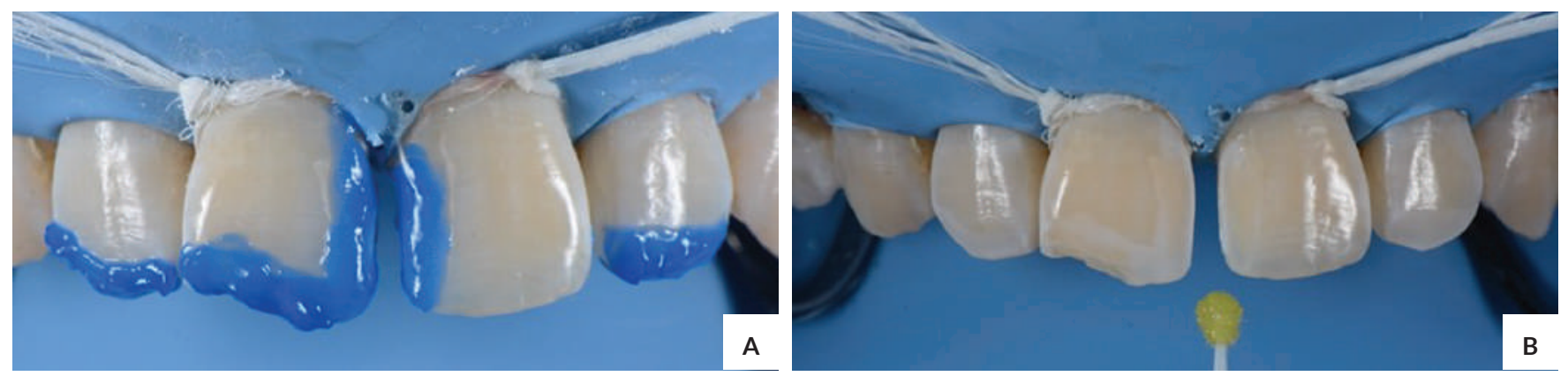

Figure 5. (A) Etching and (B) Bonding.

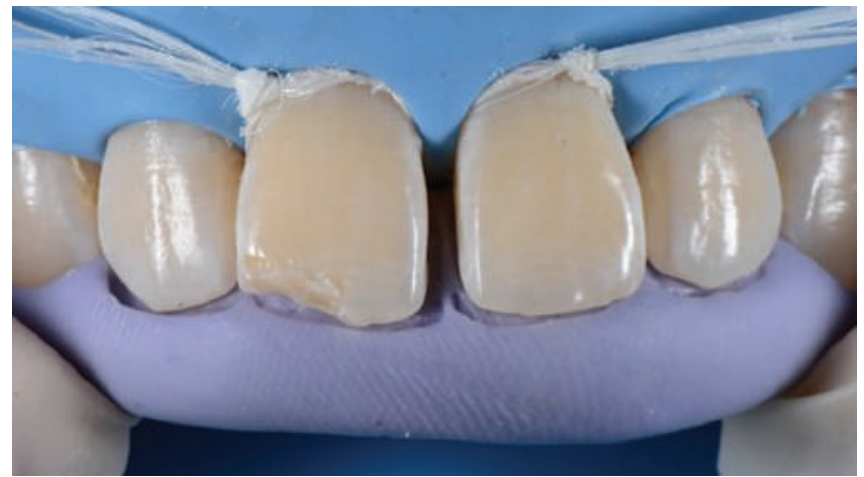

Figure 6. Palatal Guide.

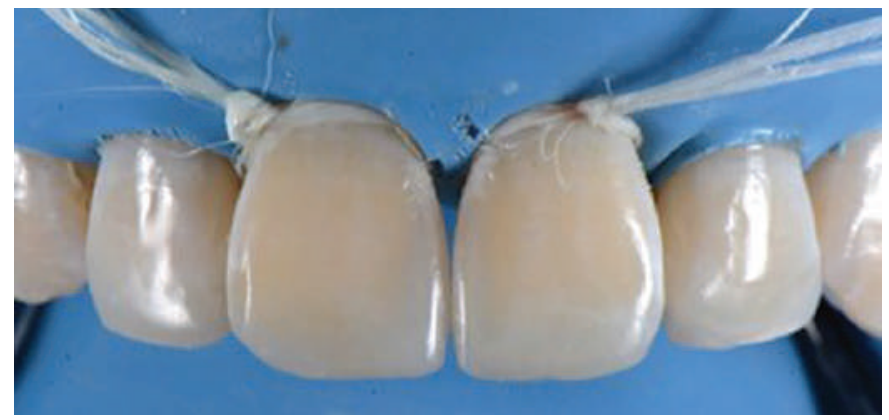

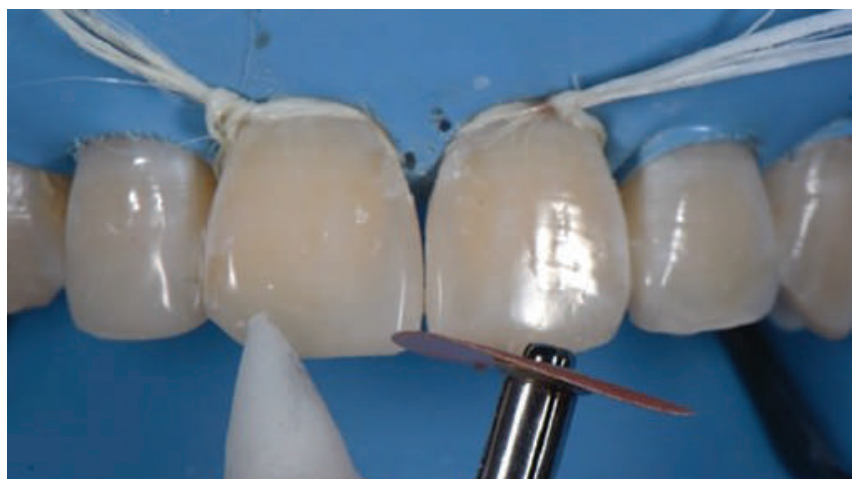

Figure 7. Polishing and Finishing.

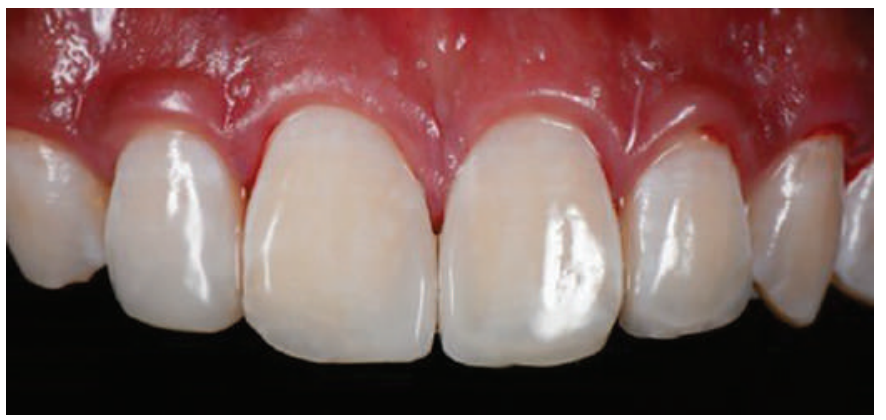

Figure 8. Final result after restoration. (A) Immediately post operative. (B) 1 week post operative.

\section{CASE 2}

A 20-year-old female patient was referred to Airlangga Dental Hospital with white spots and a small black area on the anterior upper teeth. The patient was not confident of her dental appearance, thus, avoided smiling. Examination revealed the presence of white spots on tooth \#7 and \#8. There were cavities between the lateral and maxillary central incisors (Figure 9A).

After taking the patient's informed consent, a rubber dam was placed. (Figure 9B) Shade selection was made using the button technique (Figure 10). In this case, shade UD2 (Enamel plus HRi, Micerium, Avegno, Genova, Italy) was used for the dentin and shade A1 (Palfique LX5, Tokuyama Dental, Tokyo, Japan) for the enamel.
All the white spots and caries were removed and a long bevel with diamond bur was placed on the labial surface. (Figure 11).

After etching the tooth surfaces with $37 \%$ phosphoric acid (Figure 12A), Universal Adhesive Prime\&Bond universal $^{\mathrm{TM}}$ was applied. Excess solvent was evaporated with a gentle blow of air using a syringe. A bonding agent was applied and photocured for 20 seconds. (Figure 12B)

Layering techniques of composite were carried out to achieve a natural color blend. Shade UD3 dentin (Enamel plus HRi, Micerium, Avegno, Genova, Italy) was used to build the dentin on tooth \#8. To achieve a translucent effect, shade A2 (Palfique LX5, Tokuyama Dental, Tokyo, Japan) was chosen to imitate the optical property of the enamel. (Figure 13A) Restorations were textured and polished using an abrasive disc and Enhance ${ }^{\circledR} \mathrm{PoGo}^{\circledR}$ system. (Figure 13B) 

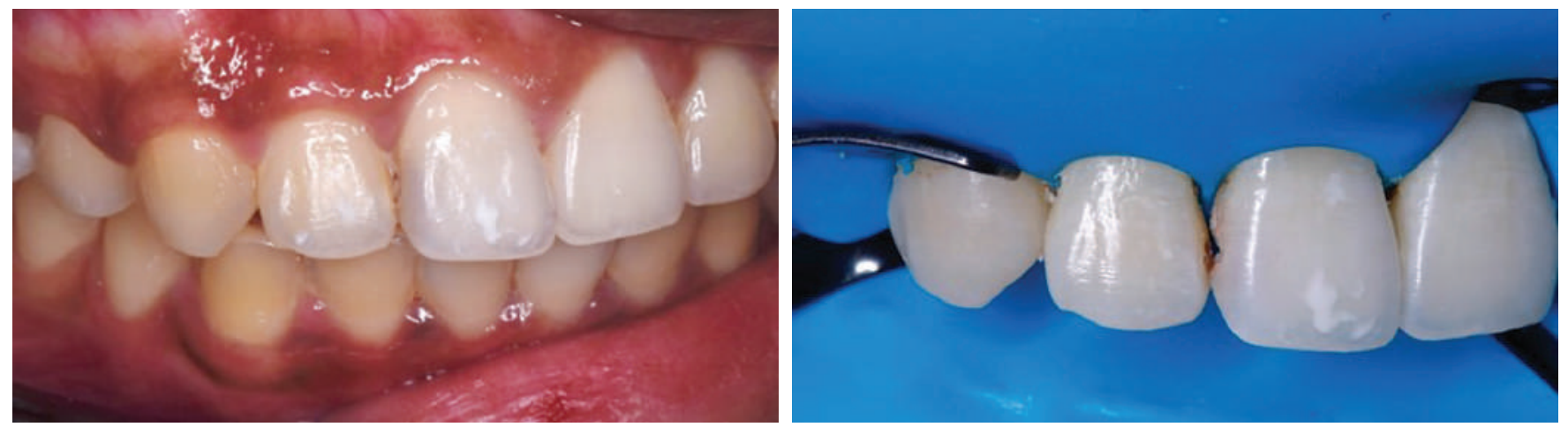

Figure 9. (A) Preoperative look. (B) Rubberdam insertion.

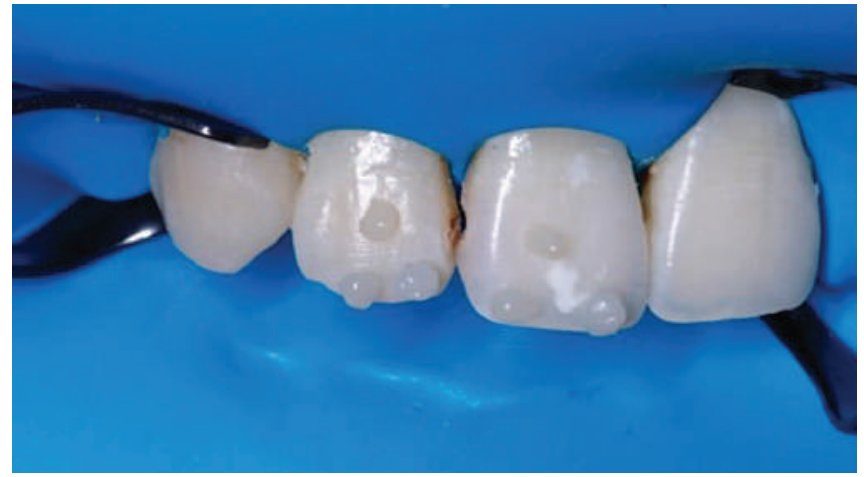

Figure 10. Composite Shades Selection.
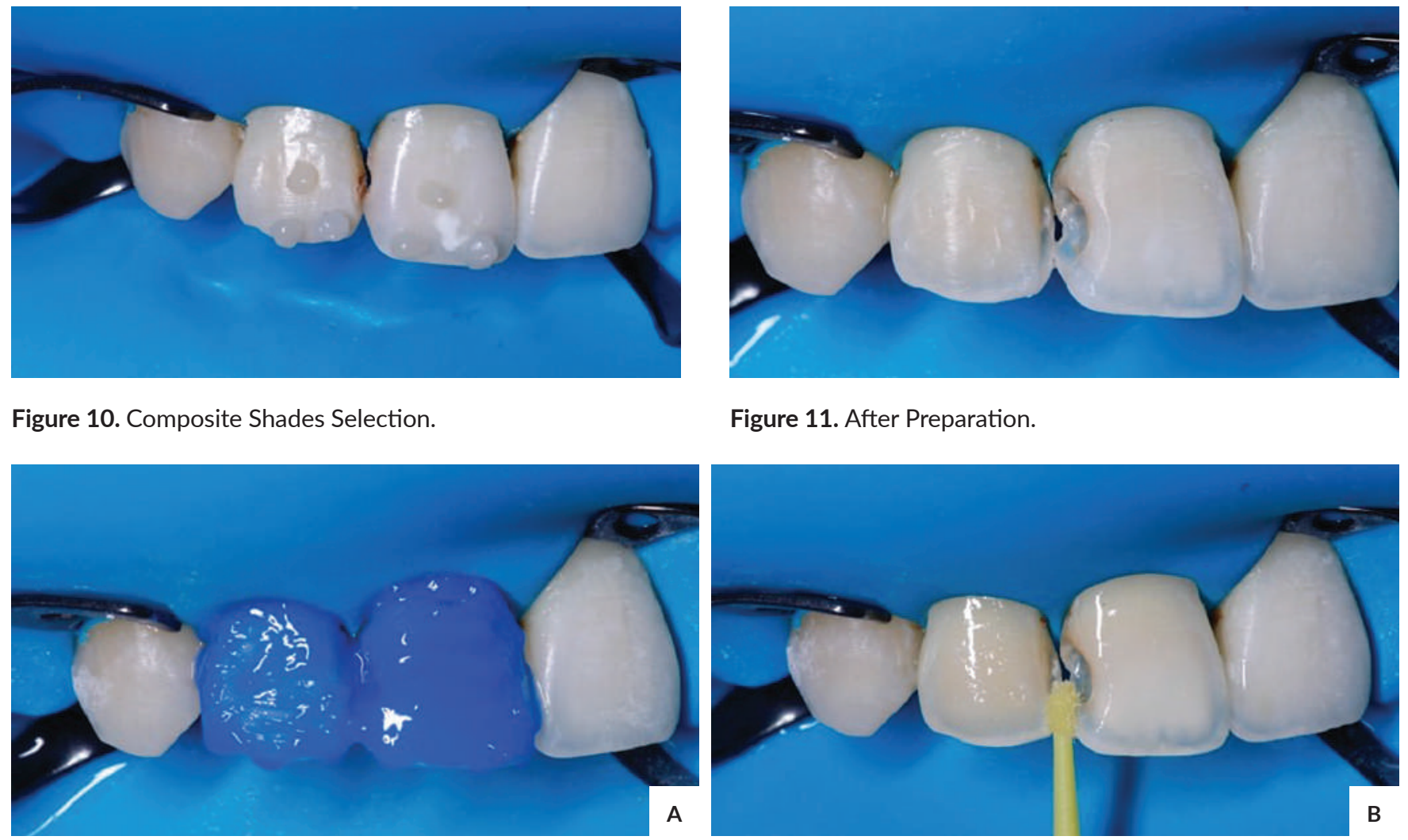

Figure 11. After Preparation.

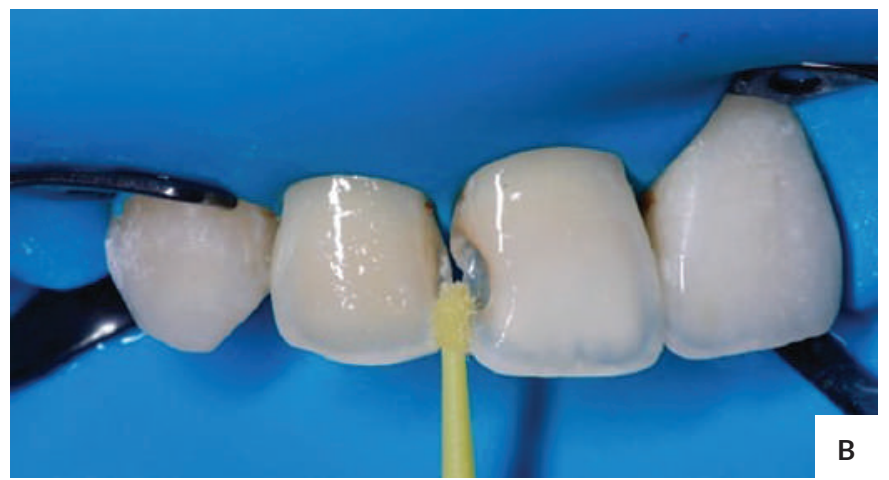

Figure 12. (A) Etching and (B) Bonding.
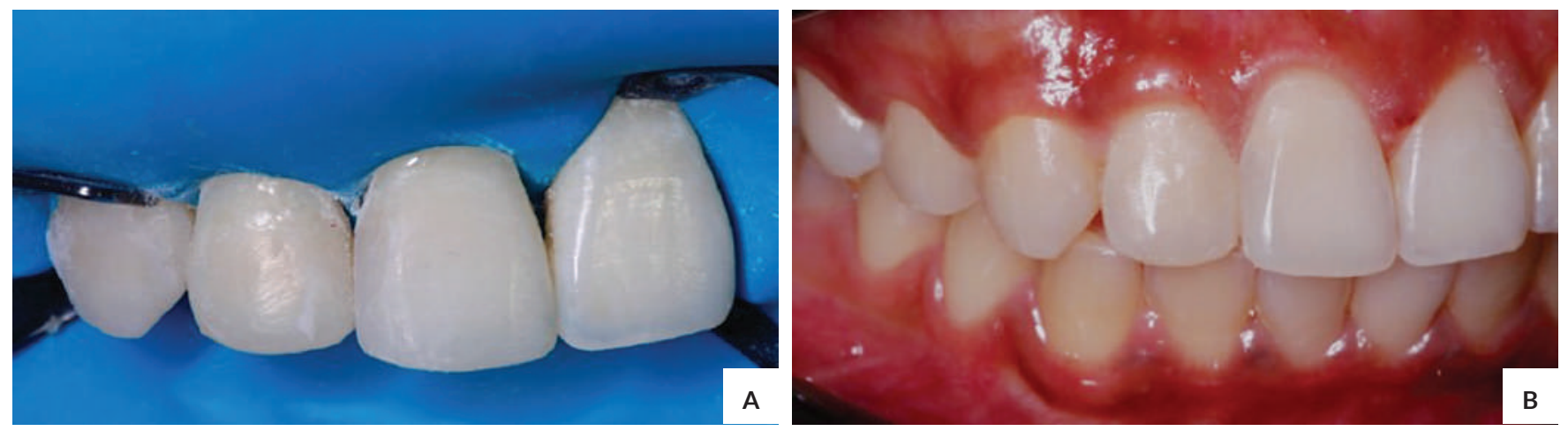

Figure 13. (A) Before Polishing and Finishing (B) Final Result. 


\section{DISCUSSION}

Researchers have found that the judgements an individual makes concerning the personal characteristics of others are influenced by dental appearance. It is widely accepted that dental esthetics also contribute to a person's facial, physical attractiveness, health, and beauty. Dental esthetics is essential in the overall physical attractiveness, with smile esthetics now receiving the attention of dental professionals recently. Adults with increased overjet, crowding, or deep bites, and adolescents with excessive display of anterior teeth, have been reported to have significantly lower self-esteem ratings and with a greater negative psychosocial impacts than those without dental problems. ${ }^{4}$ Twigge et al (2016) showed that $85 \%$ of adolescents expect to have better dental appearance. ${ }^{5}$

A recent study showed that both facial and bodily attractiveness are predictive of overall attractiveness with the face emerging as the most powerful predictor. This shows that individuals with positive personality characteristics have a face that is considered socially attractive. In addition, smile esthetics contributes significantly to facial attractiveness because during social engagements, the interlocutors initially pay attention on the eyes and on the mouth of the speaker. Therefore, smile plays a crucial role in determining facial expression and appearance. ${ }^{6}$

In case 1 , the patient had a diastema and a slightly misalignment anterior teeth. Diastema refers to a gap or excessive space between two adjacent teeth. It occurs more often in the median plane of the maxillary arch on the two adjacent central incisors, thus, leading to a midline diastema. ${ }^{7}$ Midline diastema has a multifactorial etiology in addition to the labial frenulum, microdontia, mesiodens, peg-shaped lateral incisors, lateral incisor agenesis, cysts in the midline region, and habits such as finger sucking, tongue thrusting, and/or lip sucking, dental malformations, genetics, maxillary incisor proclinations, dental skeletal discrepancies, and imperfect coalescence of the interdental septum. ${ }^{8}$ After the etiology was evaluated, a decision on the proper treatment was undertaken. ${ }^{9}$ In this case, the teeth were slightly aligned and the interdental spacing between the maxillary central incisors reduced to less than $2 \mathrm{~mm}$ using direct restoration composite. The misaligned teeth were slightly roughened with a coarse sandpaper disc to recontour the teeth rather than prepping it with a bur. This technique minimized unnecessary reduction in some facial tooth structure.

In case 2, the patient had white spots on the anterior teeth. The white spots indicated an initial carious lesion which implied that there was a subsurface area with loss of mineral beneath an intact enamel surface. ${ }^{10}$ Conventional treatments for these white spot lesions include the application of topical fluoride, improvement in the oral hygiene of the patient, and the use of remineralizing agents like ACPCPP. ${ }^{11}$ In case 2 , the patient claimed that she previously underwent bleaching and topical fluoride application, but the result was unsatisfactory. Direct restoration composite was chosen to correct the lesions.

Direct veneer treatment were indicated in both cases as it allows the evaluation and manipulation on a single treatment session.

Recent composite resin materials offer an expanded range of shades that perfectly emulate natural tooth. Direct composite veneering results in minimal invasion and maximum preservation of sound tooth structure when compared to indirect restorations. ${ }^{12}$ Esthetic and durability of composite materials have evolved remarkably over the years. Advances in resin technology and the presence of new materials have positive outcomes, resulting in reduced shrinkages, improved color stability, wear resistance, and biocompatibility. ${ }^{13}$

\section{CONCLUSION}

Self-confidence in young adolescent patients is associated with dental appearance. The perception of dental esthetics covers many factors such as tooth shape, position and color. Direct composite veneer may provide a solution in boosting self-confidence in young patients as they yield immediate results and instant gratification.

\section{Statement of Authorship}

All authors participated in data collection and analysis, and approved the final version submitted.

\section{Author Disclosure}

All authors declared no conflict of interest.

\section{Funding Source}

None.

\section{REFERENCES}

1. Demir F, Oktay E, Topcu F. Smile and dental aesthetics: a literature review. Med Sci. 2017;6(1):172-7. doi: 10.5455/ medscience.2016.05.8543

2. Garg N, Garg A, editors. Chapter 19 Esthetic Denistry. In: Textbook of Operative Dentistry, 3rd ed. Jaypee Brothers Medical Publishers; 2015

3. Ritter A V, Boushell LW, Walter R. Sturdevant's Art and Science of Operative Dentistry. Seventh Ed. St. Louis, Missouri: Elsevier; 2017. pp. 284-287.

4. Afroz S, Rathi S, Rajput G, Rahman SA. Dental esthetics and its impact on psycho-social well-being and dental self confidence: A campus based survey of north indian university students. J Indian Prosthodont Soc. 2013;13(4):455-60.

5. Twigge E, Roberts RM, Jamieson L, Dreyer CW, Sampson WJ. The psycho-social impact of malocclusions and treatment expectations of adolescent orthodontic patients. Eur J Orthod. 2016 Dec;38(6):593-601.

6. Scarborough L. Self-perception of Smile Aesthetics and Self-esteem [thesis]. Virginia Commonwealth University; 2011.

7. Abu-Hussein M, Watted N, Abdulgani A. An interdisciplinary approach for improved esthetic resuslts in the anterior maxilla diastema. IOSR Journal of Dental and Medical Sciences. 2015; 14(12):96-101. 
8. Korkut B, Yanikoglu F, Tagtekin D. Direct midline diastema closure with composite layering technique: a one-year follow-up. Case Rep Dent. 2016; 2016:6810984.

9. Goyal A, Nikhil V, Singh R. Diastema closure in anterior teeth using a posterior matrix. Case Rep Dent. 2016; 2016:2538526.

10. Roopa KB, Pathak S, Poornima P, Neena IE. White spot lesions: A literature review. J Pediatr Dent. 2015; 3(1):1-7.

11. Gugnani N, Pandit IK, Gupta M, Josan R. Caries infiltration of noncavitated white spot lesions: A novel approach for immediate esthetic improvement. Contemp Clin Dent. 2012; 3(Suppl 2): S199-S202. doi:10.40103/0976-237X.101092.
12. Newton JT, Prabhu N, Robinson PG. The impact of dental appearance on the appraisal of personal characteristics. Int J Prosthodont. 2003; 16(4):429-34.

13. Sowmya K, Dwijendra KS, Pranitha V, Roy KK. Esthetic Rehabilitation with Direct Composite Veneering: A Report of 2 Cases. Case Rep Dent. 2017; 2017:7638153.

\section{The Acta Medica Philippina is now accepting} original scientific papers, review articles and case reports for its upcoming issues. Please follow the format for submission as indicated in the "Instructions to Authors" elsewhere in the journal. All papers received shall be properly acknowledged. For inquiries and submission of proposals, please email us at actamedicaphilippina.upm@up.edu.ph 